OPEN ACCESS

Edited by:

William Cho,

Queen Elizabeth Hospital, Hong Kong

Reviewed by:

Yuriy L. Orlov,

First Moscow State Medical

University, Russia

Argyris Papantonis,

University Medical Center Göttingen,

Germany

*Correspondence:

$\mathrm{Ji}$-Fan Hu

jifan@stanford.edu

Wei Li

jdyylw@163.com

${ }^{\dagger}$ These authors have contributed equally to this work

Specialty section:

This article was submitted to

RNA,

a section of the journal

Frontiers in Genetics

Received: 06 December 2019

Accepted: 09 March 2020

Published: 31 March 2020

Citation:

Chen J, Wang Y, Wang C, Hu J-F and Li W (2020) LnCRNA Functions as

a New Emerging Epigenetic Factor

in Determining the Fate of Stem Cells.

Front. Genet. 11:277.

doi: $10.3389 /$ fgene.2020.00277

\section{LncRNA Functions as a New Emerging Epigenetic Factor in Determining the Fate of Stem Cells}

\author{
Jingcheng Chen ${ }^{1,2+}$, Yizhuo Wang ${ }^{1 \dagger}$, Cong Wang ${ }^{1,2}$, Ji-Fan Hu' ${ }^{1,2 *}$ and Wei $\mathrm{Li}^{1 *}$ \\ ${ }^{1}$ Cancer Center, The First Hospital of Jilin University, Changchun, China, ${ }^{2}$ VA Palo Alto Health Care System, Stanford \\ Medical School, Stanford University, Palo Alto, CA, United States
}

Pluripotent stem cells have broad applications in regenerative medicine and offer ideal models for understanding the biological process of embryonic development and specific diseases. Studies suggest that the self-renewal and multi-lineage differentiation of stem cells are regulated by a complex network consisting of transcription factors, chromatin regulators, signaling factors, and non-coding RNAs. It is of great interest to identify RNA regulatory factors that determine the fate of stem cells. Long non-coding RNA (IncRNA), a class of non-coding RNAs with more than 200 bp in length, has been shown to act as essential epigenetic regulators of stem cell pluripotency and specific lineage commitment. In this review, we focus on recent research progress related to the function and epigenetic mechanisms of IncRNA in determining the fate of stem cells, particularly pluripotency maintenance and lineage-specific differentiation. We discuss the role of the Oct4 and Sox2 promoter-interacting IncRNA as identified by Chromatin RNA In Situ reverse Transcription sequencing (CRIST-seq). Further understanding of their potential actions will provide a basis for the development of regenerative medicine for clinical application. This work offers comprehensive details and better understanding of the role of IncRNA in determining the fate of stem cells and paves the way for clinical stem cell applications.

Keywords: long non-coding RNA, stem cell, pluripotency, cell differentiation, reprograming, epigenetics, promoter-interacting IncRNA network

\section{INTRODUCTION}

Pluripotent stem cells (PSCs), including ESCs and iPSCs, have great potential in regenerative medicine. ESCs are derived from the ICM of a mammalian blastocyst and can differentiate into different cell types to form the endoderm, mesoderm, and ectoderm (Thomson et al., 1998). On the other hand, iPSCs are induced by pluripotent reprograming of somatic cells with specific transcription factors (Takahashi and Yamanaka, 2006; Yu et al., 2007). PSCs have the ability to self-renew and differentiate into different cell types, making them ideal models for exploring embryonic development early events and understanding regenerative medicine (Borsos and TorresPadilla, 2016). It is well known that the function of PSCs is regulated by many growth factors, including LIF, BMPs, FGF4, and their receptor-related signaling (Chen C.Y. et al., 2017; Hassani et al., 2019). PSCs express characteristic transcription factors, such as Oct4, Nanog, Sox2, and Klf4. The collaboration and synergy of these transcription factors are crucial for maintaining stemness 
and pluripotency. The cell fate of PSCs is controlled or regulated by a complex process, involving transcription factors, signaling pathways, non-coding RNAs and their collaborative interaction. Hence, understanding the molecular mechanisms underlying determination of PSC fate will be of significance in manipulating their self-renewal and differentiation into specific cell lineages for potential application.

Long non-coding RNA refers to a family of non-coding RNAs with more than 200 bp in length and is transcribed by RNA polymerase II, usually $5^{\prime}$ capped, spliced and polyadenylated (Kung et al., 2013; Quinn and Chang, 2016). LncRNA can be transcribed from different genome regions, including introns, exons, intergenic connections, and other areas. Currently, there are about 30000 lncRNAs identified in human and mice (Frankish et al., 2019), but only a handful part of them have been recognized for their function. Structurally, lncRNA is less evolutionally conserved than mRNA (Ponting et al., 2009), and they can display complicated secondary architecture after interacting with proteins, DNA or RNA to form a proper tertiary structure for their functional activities (Wang and Chang, 2011). LncRNA acts as essential regulators in a variety of biological or cellular processes, including chromatin remodeling, transcription, posttranscriptional processing, intracellular trafficking, metabolism, development and differentiation (Ponting et al., 2009; Wapinski and Chang, 2011). LncRNA has the ability to regulate the self-renewal and differentiation of PSCs (Mercer et al., 2009;

Abbreviations: ADNCR, adipocyte differentiation-associated long non-coding RNA; ADSC, adipose-derived stem cell; AGIL, asymmetric G-rich internal loop; ALP, alkaline phosphatase; ANCR, anti-differentiation ncRNA; CRISTseq, chromatin RNA in situ reverse transcription sequencing; BMP, bone morphogenetic protein; BMSC, bone mesenchymal stem cell; CEBPA, CCAAT Enhancer Binding Protein $\alpha$; CeRNA, competing endogenous RNA; ChIP-Seq, chromatin immunoprecipitation with the next-generation DNA sequencing; CNBP, nucleic acid binding protein; CNS, central nervous system; CPCs, cardiac precursor cells; DANCR, differentiation antagonizing non-protein coding RNA; DLK1-DIO3, delta-like homolog 1 gene and the type III iodothyronine deiodinase gene; DPPA2, developmental pluripotency-associated 2; DRR, distal regulatory region; Dum, Dppa2 upstream binding muscle lncRNA; Dusp9, dual specificity phosphatase 9; ESC, embryonic stem cell; Fendrr, Fetal-lethal non-coding developmental regulatory RNA; FGF4, fibroblast growth factor 4; Gas5, growtharrest-specific transcript 5; hASC, human adipose-derived stem cell; HBL1, Heart Brake lncRNA 1; HBMSC, human bone marrow-derived mesenchymal stem cell; hESC, human embryonic stem cell; HnRNP-K, heterogeneous nuclear ribonucleoprotein $\mathrm{K}$; HOTAIR, HOX transcript antisense RNA; ICM, inner cell mass; IMP2, IGF2-mRNA-binding protein 2; iPSC, induced pluripotent stem cell; LIF, leukemia inhibitory factor; LncPRESS, IncRNA p53 regulated and ESC associated 1; LncRNA, long non-coding RNA; LPM, lateral plate mesoderm; Malat1, metastasis-associated lung adenocarcinoma transcript 1; MEG3, maternally expressed gene 3; mESC, mouse embryoid stem cell; MesP1, mesoderm posterior 1; MHC, myosin heavy chain; MIAT, myocardial infarctionassociated transcript; MITF, microphthalmia-associated transcription factor; MLL, mixed-lineage leukemia; MUNC, MyoD upstream ncRNA; PAX2, paired box gene 2; PMA, protein microarrays; PPAR, peroxisome proliferator-activated receptor; PRC2, Polycomb Repressive Complex 2; pRNA, promoter associated RNA; PTBP, poly pyrimidine tract-binding protein; RAT-seq, RNA reverse transcription-associated trap sequencing; REST, neuron-restricting silencing factor; RIA-seq, RNA interactome sequencing; RIP, RNA immunoprecipitation; RMST, Rhabdomyosarcoma 2-associated transcript; RNA-Seq, RNA transcriptome sequencing; RNP, ribonucleoprotein; SRA, steroid receptor RNA activator; SRCAP, Snf2 related CREBBP activator protein; TANC1, tetratricopeptide repeat, ankyrin repeat and coiled-coil containing 1; TBX2, T-box transcription factor 2; TINCR, terminal differentiation-induced ncRNA; TSS, transcription start sites; TUNA, Tcll upstream neuron-associated lncRNA; UCE, ultraconserved enhancer.
Cao, 2014). Here, we provide an updated overview on how lncRNA epigenetically regulates PSC function.

\section{THE ROLE OF LnCRNA IN STEM CELL PLURIPOTENCY}

Stem cell pluripotency and differentiation are regulated by a multilayered and complicated network, including lncRNA. Since PSCs hold therapeutic potential in many types of diseases, understanding the regulatory network for maintenance of stem cell pluripotency will be essential for manipulating PSCs in clinical applications. Previous studies have demonstrated that IncRNAs are crucial regulators for regulating stem cell pluripotency (Chen and Zhang, 2016; Rosa and Ballarino, 2016). Transcriptional analysis of human fibroblasts, their derivative iPSCs, and ESCs showed that 133 lncRNAs were up-regulated and 104 lncRNAs were down-regulated in iPSCs and ESCs as compared with fibroblasts (Loewer et al., 2010). The upregulated lncRNAs may bind to Oct4, Sox 2 and Nanog in iPSCs, indicating that they are crucial for maintenance of stem cell pluripotency. In a second study, more than 130 lncRNAs were examined by shRNA knockdown for their function in the of ESC pluripotency maintenance (Guttman et al., 2011). ChIP-Seq was performed to generate a database to illustrate the relationship between transcription factors and lncRNAs in pluripotency regulation (Yang et al., 2013). Most lncRNAs were regulated by key pluripotent transcription factors, including Oct4, Sox2 and Nanog (Ng et al., 2012; Fico et al., 2019). Recently, Du and coauthors identified multiple pluripotency-associated lncRNAs embedded in the chromatin regulatory network by combining RNA-Seq and RAT-seq (Du et al., 2018).

The intracellular localization of a lncRNA provides a clue for its action mode (Batista and Chang, 2013). Nuclear lncRNAs often interact with chromatin modification factors, RNA binding proteins or transcription factors to regulate gene expression (Figure 1). The lncRNA Gm15055 is highly induced by Oct4 and is located near the HOX gene cluster of mESCs. Gm15055 can interact with and recruit PRC2 to maintain H3K27me3 levels on HoxA genes (Liu et al., 2016). The expression of lncRNA p53regulated and ESC-associated 1 (LncPRESS1) is suppressed by p53 (Jain et al., 2016), and its knockdown induces spontaneous differentiation in hESCs by down-regulating OCT4, NANOG and $c-M Y C$, but it up-regulates HOXA2, HOXB1, and FOXA2 expression. Mechanistically, IncPRESS1 can interact with SIRT6, inhibiting SIRT6 attachment to chromatin and maintaining the histone $\mathrm{H} 3 \mathrm{~K} 56$ and $\mathrm{H} 3 \mathrm{~K} 9$ promoter acetylation to safeguard

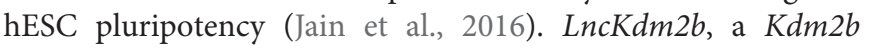
divergent lncRNA, is a positive regulator of ESC self-renewal and early embryogenesis. It recruits Snf2-related CREBBP activator protein (SRCAP)-contained remodeling complex to the $Z b t b 3$ promoter and activates $Z b t b 3$ expression to induce Nanog expression (Ye et al., 2018). Another study reports that lncRNA-ES1 (AK056826), lncRNA-ES2 (EF565083) and lncRNAES3 (BC026300) are differentially expressed in hESCs nuclei, and they can bind near the TSS of the OCT4 and NANOG promoters to enhance hESC pluripotency (Ng et al., 2012). Their 


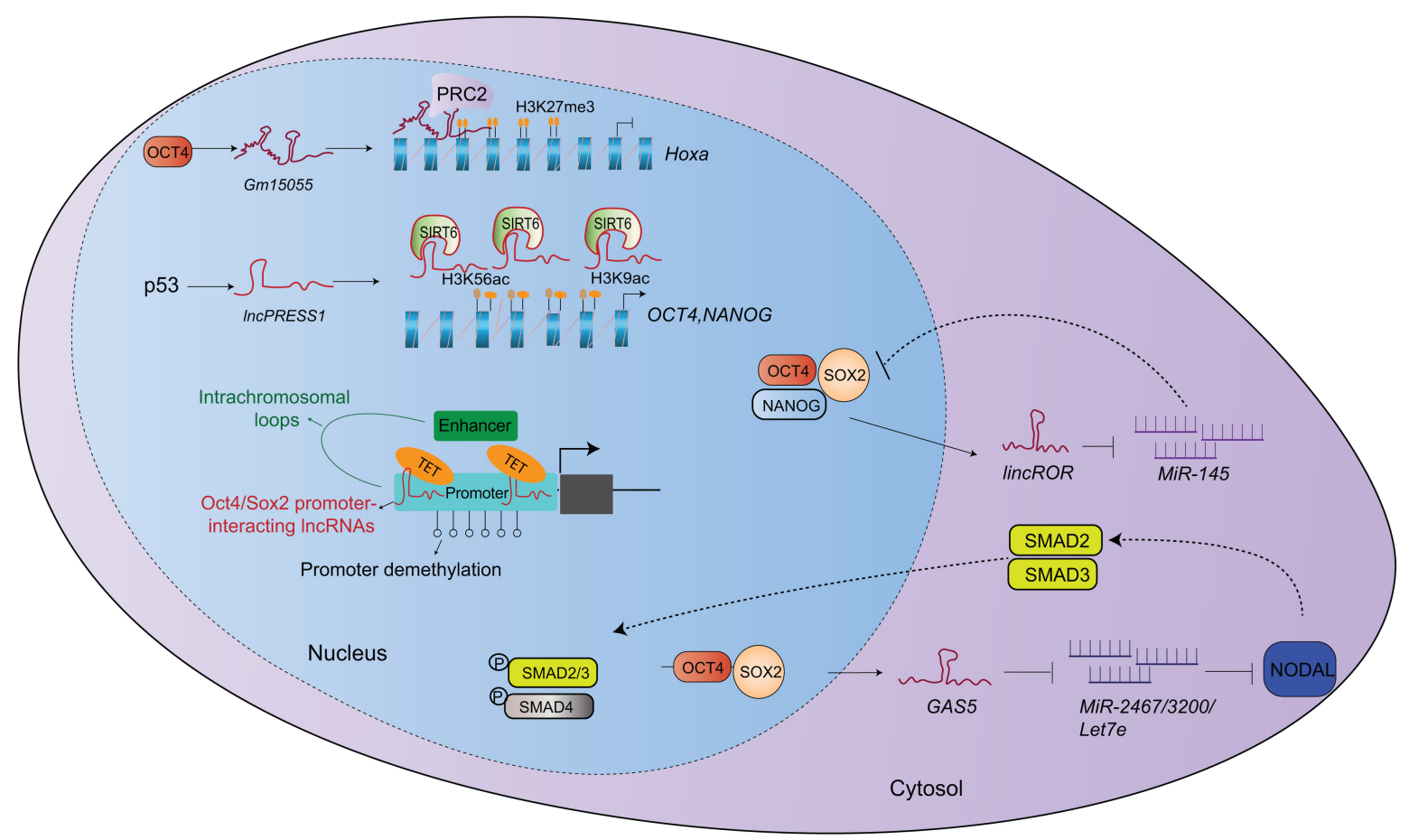

FIGURE 1 | Mechanisms of IncRNA in regulating stem cell pluripotency. Nuclear IncRNAs regulate the expression of pluripotent genes via chromatin remodeling. While IncRNAs in cytosol mainly act as post-transcriptional regulators. They work as miRNA sponges to affect gene expression or signaling pathway.

deficiency reduces the pluripotency-related gene expression, but enhances the neuroectoderm, endoderm and mesoderm germ layer-related gene expression. LncRNA-ES1 and IncRNAES2 physically interact with SUZ12 and SOX2, and they act as modular scaffolds for SUZ12 (or PRC2) and SOX2 in hESCs. SOX2 may induce H3K27 methylation during the neuroectoderm lineage commitment, leading to gene silencing at these loci. Furthermore, SOX2 binds lncRNAs to prevent the binding of other core pluripotency-associated transcription factors. LncRNA-ES3 silencing displays a phenotype similar to NANOG knockdown in hESCs (Ng et al., 2012). Zeb2-NAT is a Zeb2 antisense lncRNA that can be upregulated in fibroblasts with aging. Zeb2-NAT deficiency increases the reprograming efficiency of aged fibroblasts into iPSCs and maintains ESC selfrenewal and pluripotency. Hence, Zeb2-NAT is an early marker for pluripotency loss (Bernardes de Jesus et al., 2018).

Cytoplasmic lncRNAs are functionally different from nuclear lncRNAs, and mainly act as post-transcriptional regulators by modulating mRNA degradation and protein translation (Figure 1). By comparing ESCs and fibroblasts, Loewer et al. identified 28 "iPSC-enriched" lncRNAs. Among them, linc-RoR regulated reprograming efficiency by binding to OCT4, SOX2, and NANOG (Loewer et al., 2010). Further study revealed that linc-RoR acted as a ceRNA and sponges miRNA-145 to enhance OCT4, SOX2, and NANOG expression and self-renewal in iPSCs (Wang et al., 2013). The lncRNA GAS5, a tumor suppressor, is highly expressed in hESC cytoplasm and is induced by OCT4 and SOX2. Knockdown of GAS5 results in stem cell differentiation by reducing hESCs in the $\mathrm{G} 2 / \mathrm{M}$ phase, indicating that GAS5 promotes hESC self-renewal cells. GAS5 can sponge $m i R$ 2467-5p, miR-3200-3p and let-7a/e-5p to enhance the NODAL signaling (Xu et al., 2016). The Cyrano lncRNA is located in ESC cytoplasm and nuclei. It directly interacts with $m i R-7$ to enhance Nanog expression and ESC self-renewal (Smith et al., 2017). Cyrano silencing inhibits ESC self-renewal and survival, and it downregulates Nanog expression (Smith et al., 2017). The lincU lncRNA is highly expressed in the cytoplasm of naïve state stem cells in a Nanog-dependent manner. LincU can stabilize Dusp9 from ubiquitination proteasome-mediated degradation to inhibit the MAPK/ERK signaling, but it enhances mESC pluripotency (Jiapaer et al., 2018).

Collectively, although lncRNAs regulate the pluripotency by varying mechanisms, they center on the stemness-related Oct4, Sox2 and Nanog expression and protein stabilization in PSCs. Regulation of these transcription factors is crucial for self-renewal of different PSC types.

\section{LnCRNA ACTS AS A NOVEL CLASS OF CHROMATIN RNA REGULATORY FACTOR IN PLURIPOTENT REPROGRAMING}

Terminally differentiated somatic cells can be reprogramed to pluripotent status as iPSCs by ectopic expression of defined factors. However, this reprograming process is time-consuming and extremely inefficient, which hinders 
potential clinical applications for regenerative medicine. To explore the mechanisms underlying reprograming, Zhang and coauthors used the chromatin conformation capture (3C) approach to compare promoter DNA binding and chromatin architecture between iPSCs and the "non-iPSCs" that expressed lentiviral reprograming-initiating factors, but failed to complete reprograming to pluripotency. It is interesting to note that the formation of a promoter-enhancer intrachromosomal loop architecture is a critical epigenetic barrier that must be overcome for the successful pluripotency induction (Zhang et al., 2013; $\mathrm{Hu}$ and Hoffman, 2014). The non-iPSCs could not achieve pluripotency, partially due to the lack of this intrachromosomal looping (Zhang et al., 2013; Hu and Hoffman, 2014). Thus, it is critical to identify molecular factors that orchestrate this pluripotency-specific intrachromosomal loop.

Long non-coding RNA consists of essential components of the three-dimensional genome structure. Several previous studies have identified lncRNAs that mediate the formation gene regulatory chromosome loops (Zhang et al., 2014; Pisignano et al., 2019). Functionally, these chromatin loop structures may bring distant enhancer elements close to the core promoter for optimal gene expression (Zhang et al., 2013; Wang $\mathrm{H}$. et al., 2015). Recently, Zhang et al. (2019) reported to the CRIST-seq approach to map lncRNA within regulatory elements of stemness genes (Figure 2). They profiled the lncRNA interaction network in the Sox 2 and Oct4 promoters using this approach. Among the identified lncRNAs, Snhg14 was abundantly expressed in iPSCs and ESCs. Functional assays confirmed that Snhg14 was required to maintain stem cell pluripotency. Jia et al. (2020) later used CRIST-seq/RNAseq to identify Oplr16 (Oct4 promoter-interacting LncRNA 16) as another pluripotency-associated chromatin RNA factor. Oplr16 enhanced reprograming epigenetically by coordinating intrachromosomal looping and DNA methylation in the Oct4 promoter. Wang et al. (2020) used CRIST-seq to identify Peblr20 ( Pou5F1 enhancer binding $\underline{\text { lncRNA }}$ 20) as another reprogramingassociated lncRNA. Notably, Peblr20 binds to the Oct4 enhancers, where it recruits TET2 demethylase and activates the enhancer RNA (eRNA) pathway. Thus, Peblr20 functions as a critical pluripotent lncRNA that utilizes a novel trans epigenetic eRNA mechanism to control stem cell fate. Exploration of the genomewide binding sites of central transcription factors and the lncRNA interactome during cell fate conversion may promote comprehensive understanding of the transcription network in reprograming.

\section{LncRNA AS A PROMISING EPIGENETIC REGULATOR OF STEM CELL DIFFERENTIATION AND SPECIFIC CELL LINEAGE COMMITMENT}

Pluripotent stem cells can differentiate and commit to a specific lineage in a given environment. The acquisition of cellular identities is a well-organized, precisely timed, and robustly executed process. The lack of effective differentiation and accurate specification of cell types hindered the widespread use of stem cells. Lineage differentiation of stem cells is regulated by a diverse and complex system that includes lncRNA. We will discuss the role of IncRNA in cell lineage commitment and focus on how lncRNA regulates PSC differentiation into adipocytes, osteogenic, muscle, cardiomyocytes, neurons, skin, and blood cells.

\section{LnCRNA IN THE ADIPOGENIC DIFFERENTIATION OF STEM CELLS}

Long non-coding RNA is involved in the adipogenic differentiation of stem cells. Understanding the mechanisms underlying adipogenesis regulation by lncRNA may reveal new therapeutic targets to combat obesity and its related diseases (Tye et al., 2015).

Long non-coding RNA $S R A$ is the first lncRNA identified for functional adipogenesis promotion (Lanz et al., 2003). SRA can enhance the expression of $\operatorname{PPAR} \gamma$, a main adipogenesis regulator. $S R A$ silencing inhibits the differentiation of preadipocytes 3T3-L1 cells. Furthermore, SRA can enhance insulin sensitivity by promoting cell cycling, inhibiting inflammatory gene expression and the TNFa-induced phosphorylation of c-Jun NH2-termianl kinase in pre-adipocytes. $S R A^{-/-}$mice are lean with reduced adipogenic gene expression (Sheng et al., 2018). The lncRNA adipogenic differentiation-induced noncoding RNA (ADINR) is induced by adipogenesis and positively regulates both PPAR $\gamma$ and CEBPA expression by recruiting PA1 (a component of the MLL3/4 complex) to the CEBPA promoter and impacting $\mathrm{H} 3 \mathrm{~K} 4 \mathrm{me} 3$ and $\mathrm{H} 3 \mathrm{~K} 27 \mathrm{me} 3$ histone modification (Xiao et al., 2015). In addition, lncRNA Blnc1 can promote adipocyte differentiation with essential transcription factors Ucp1, PPAR $\gamma$ and Ebf2. Functionally, Blnc1 can interact with and recruit Ebf2 to enhance Ucp1 expression and mitochondrial respiration-associated adipogenic differentiation (Zhao et al., 2014).

Maternally expressed gene 3 was identified as an osteogenic differentiation-related lncRNA in BMSCs (Zhuang et al., 2015). A recent study has shown that MEG3 can inhibit adipogenic differentiation by sponging miR-140-5p because MEG3 silencing enhances adipogenic differentiation in pre-adipocytes ( $\mathrm{Li} \mathrm{Z}$. et al., 2017). It is well known that miR-140-5p can enhance adipogenic differentiation by upregulating PPAR $\gamma$ and CEBPA expression, two principal adipogenic transcription factors ( $\mathrm{Li}$ Z. et al., 2017). Furthermore, ADNCR is an inhibitory lncRNA of adipogenic differentiation that functions as a ceRNA for miR-204 to promote the miR-204-targeted SIRT1 expression, given that SIRT1 can inhibit adipocyte differentiation by interacting with NCoR and SMART to suppress PPAR $\gamma$ activity. Hence, ADNCR can enhance the adipogenesis inhibitory SIRT1 expression in a miR-204-dependent manner (Li et al., 2016). Thus, lncRNA can regulate adipogenesis by directly interacting with chromatin modification complexes or transcription factors. LncRNA can also act as ceRNAs for miRNAs to influence adipogenesis regulator expression. These lncRNAs form a network to regulate the dynamic adipogenesis process. However, 

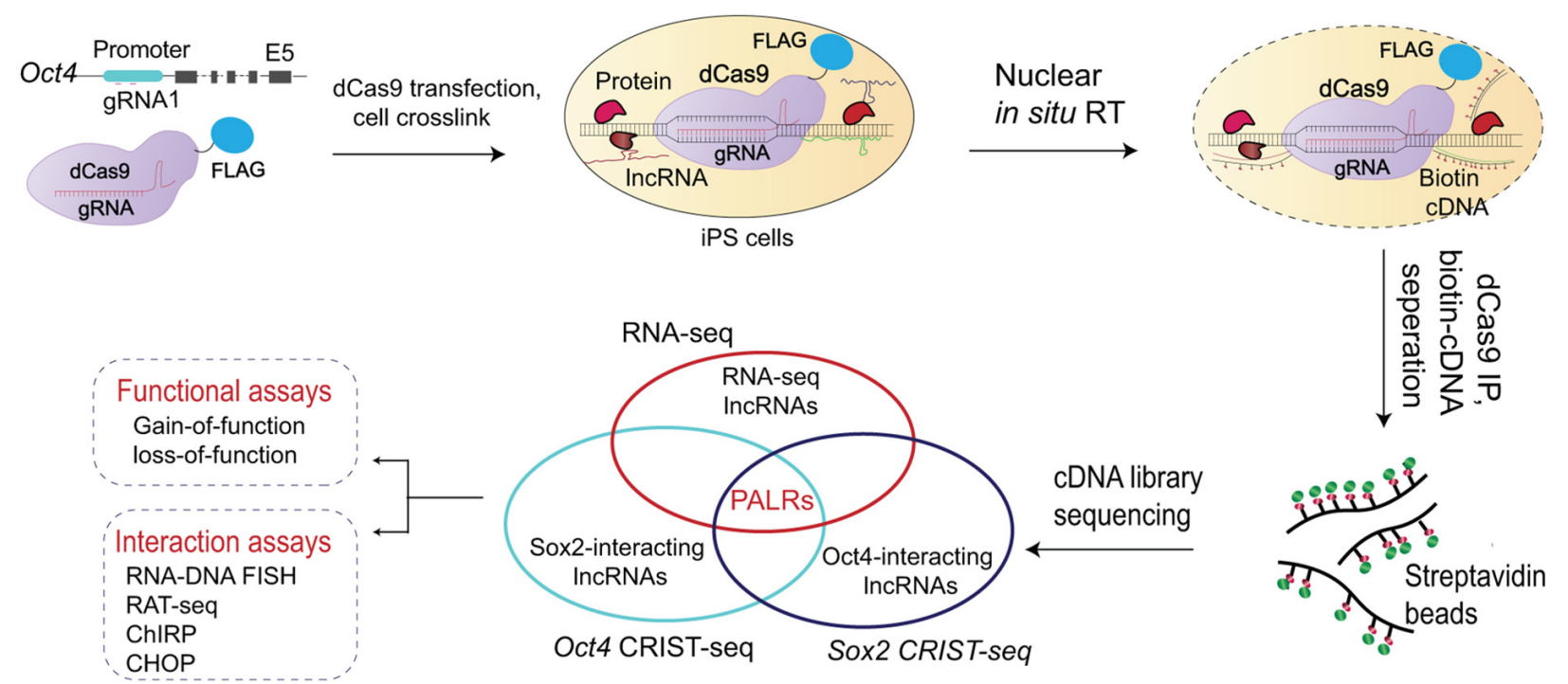

PALRs: pluripotency-associated IncRNAs

FIGURE 2 | Mapping stemness gene promoter-interacting IncRNA by CRIST-seq. The CRIST-seq assay combines the specificity of Cas9 gene targeting with the simplicity of biotin-IncRNA labeling. Target cells are first transfected with lentiviruses carrying dCas9-FLAG/gRNA that target the promoter of stemness genes, like Oct4 and Sox2, that are two core stem cell factors essential for the establishment and maintenance of pluripotency. The cells are crosslinked to fix the promoter chromatin DNA-IncRNA complex. Following lysis of the cell membrane, the promoter-interacting RNAs are reverse transcribed in situ into biotin-cDNAs (cDNAs) in the isolated nuclei with biotin-dCTP. The promoter chromatin-cDNA complex is immunoprecipitated by a Cas9-FLAG antibody and the promoter-interacting biotin-cDNAs are separated by the biotin-streptavidin bead purification. The CRIST-captured cDNAs are used for library construction and Illumina sequencing is performed for identifying the IncRNAs that interact with the promoter of stemness genes. Integration of the Oct4-Sox2 CRIST-seq data with the RNA-seq data allows the identification of IncRNAs that not only interact with core factor promoters of stem cells, but are also differentially expressed during reprograming. The IncRNA-promoter interaction is subsequently validated by other tools, including RNA-DNA FISH, RAT, ChIRP, and CHOP assays. Gain- and loss-of-function assays are then used to characterize the role of identified IncRNAs in stem cells. The CRIST-seq method offers a more efficient strategy for exploring the IncRNA network in the regulation of reprograming and pluripotency.

it is still unclear how these lncRNAs interact to maintain adipogenesis homeostasis.

\section{THE ROLE OF LnCRNA IN OSTEOGENIC DIFFERENTIATION}

Bone mesenchymal stem cells can differentiate into osteoblasts, chrondrocytes, and osteocytes in osteogenic differentiation for bone tissue formation. Osteogenic differentiation stimulates ALP expression and calcium deposition. Osteogenic differentiation is stimulated and regulated by genetic and environmental factors, including lncRNA. Its deficiency can cause osteogenesis imperfecta in young children and osteoporosis, a common disease affecting many people, particularly for the elderly people and postmenopausal women in the world. Hence, understanding lncRNA regulatory roles in osteogenesis may reveal potential therapeutic targets for intervention of osteogenesis deficient diseases (Long, 2011).

Long non-coding RNA can act as miRNA spongers to modulate the miRNA-targeted gene expression (Salmena et al., 2011). Human BMSC osteogenesis induce H19 and linc-ROR lncRNAs. $H 19$ can upregulate osteogenic related gene expression and promote bone formation in vivo by targeting $m i R-141$ and $m i R-22$, two potent inhibitors of osteogenesis. These
miRNAs can down-regulate $\beta$-catenin expression, attenuating the $\mathrm{Wnt} / \beta$-catenin signaling for osteoblast development. The action of $\mathrm{H} 19$ is counteracted by its encoded $m i R-675-5 p$ that inhibits osteoblast differentiation (Liang et al., 2016). Accordingly, H19 can enhance osteogenesis by targeting $m i R-141$ and $m i R-22$ to avoid their inhibition of the Wnt/ $\beta$-catenin signaling. Similarly, linc-ROR can also enhance the expression of osteogenic genes by sponging $m i R-138$ and $m i R-145$ to enhance ZEB2 expression and downstream Wnt/b-catenin signaling eventually (Feng L. et al., 2018). LncRNA-MEG3 and IncRNA-AK141205 can enhance osteogenesis by dissociating SOX2 from the BMP4 promoter to induce BMP4 expression and by positively promoting CXCL13 expression, respectively (Zhuang et al., 2015).

On the other hand, osteogenic differentiation of hASCs downregulates lncRNA MIAT expression. This, together with the fact that MIAT deficiency promotes osteogenic differentiation in vitro and accelerates bone formation in vivo, indicates that MIAT inhibits osteogenic differentiation. MIAT silencing reverses the TNFa-inhibited osteogenic differentiation. Mechanistically, MIAT can sponge $m i R-150-5 p$ and interact with AKT to decrease oxidative stress and inflammatory factor stimulation (Shen et al., 2016; Jin et al., 2017). Similarly, osteogenic differentiation of HBMSCs down-regulates the expression of lncRNA DANCR, indicating that $D A N C R$ is an inhibitor. Indeed, DANCR deficiency elevates ALP and osteogenic marker gene expression, 
enhances cell cycling in the S phase, and DANCR over-expression has opposite effects (Zhang et al., 2018). Mechanistically, DANCR inhibits HBMSC osteogenic differentiation by targeting the p38 MAPK signaling, which can promote HBMSC differentiation, mineralization and proliferation (Zhang et al., 2018). In addition, osteogenic differentiation of BMSCs can also down-regulate lncRNA brain-derived neurotrophic factor-antisense transcript (BDNF-AS) and MIR31HG expression. BDNF-AS is transcribed from the $B D N F$ antisense, while $M I R 31 H G$ is from chromosome 9 and can be induced by the activated NF-kB. BDNF-AS can inhibit osteogenesis possibly via inverse regulation on $B D N F$ and other osteogenic signaling (Feng X. et al., 2018). MIR31HG downregulation dramatically promotes osteogenic differentiation and significantly overcomes the osteogenesis inhibition induced by hASC inflammation. MIR31HG can interact with the NF$\kappa \mathrm{B}$ to inhibit bone formation by binding to $\mathrm{I} \kappa \mathrm{Ba}$ to insult

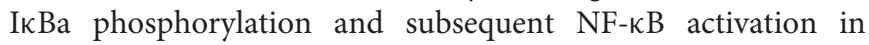
hASC. Hence, MIR31HG and NF- $\mathrm{BB}$ form a regulatory loop to improve osteogenesis efficiency in hASCs under inflammatory microenvironment. MIR31HG may be a therapeutic target for inhibiting inflammation and enhancing bone formation (Jin et al., 2016). Moreover, lncRNA ANCR is crucial for maintaining the undifferentiated cell state in human epidermis. A recent study reveals that $A N C R$ silencing promotes osteoblast differentiation because $A N C R$ can directly interact with EZH2 to catalyze $\mathrm{H} 3 \mathrm{~K} 27 \mathrm{me} 3$ in the Runx2 promoter, inhibiting Runx2 expression (Zhu and $\mathrm{Xu}, 2013$ ).

\section{LnCRNA IN MYOGENESIS}

Myogenesis is a highly ordered process that can be divided into several steps, including muscle stem cell activation, myoblast proliferation and differentiation, and myotubular formation. The process occurs in both postnatal development and the skeletal muscular regeneration after injury. It is orchestrated by a complex network involving in epigenetic regulators, transcription factors and lncRNA.

Linc-MD1, a muscle-specific lncRNA, is expressed in the cytoplasm of skeletal muscle cells and activated during myoblast differentiation. Linc-MD1 can promote myogenesis by sponging $m i R-133$ and $m i R-135$ to enhance the expression of MAML1 and $\mathrm{MEF} 2 \mathrm{C}$, which are critical transcription factors to induce muscle-specific gene expression (Cesana et al., 2011). During the embryoid development, lncRNA H19 expression is upregulated in maternal embryonic tissues, but it down-regulated after birth only in the skeletal muscles. H19 expression is strongly induced during myoblast differentiation while H19 deficiency inhibits skeletal muscule differentiation. $H 19$ exon 1 encodes $m i R$ 675-3p and miR-675-5p, which can be induced during skeletal muscule differentiation. These two microRNAs can target the transcription factor Smad to impair BMP signaling and Cdc6 expression (Dey et al., 2014b). Besides, H19 can also enhance Igf2 expression, a myogenesis stimulator in myoblasts (Kallen et al., 2013) and block the SIRT1/FoxO1 signaling (Xu et al., 2017). Dum, is induced by MyoD when myoblast differentiation begins and exclusively expressed in active myogenesis, but not in mature muscle homeostasis. Dum silencing dramatically delays myogenic differentiation. Mechanistically, the Dum gene is near Dppa2, a positive regulator of the pluripotency factor Oct4. Dum can cis-promote myogenesis to inhibit Dppa2 expression by interacting and recruiting Dnmt to $\mathrm{CpG}$ islands in the Dppa2 promoter (Wang L. et al., 2015). MyoD is crucial for myoblasts development and function. $M y o D$ controls early myogenesis by inducing myoblast cell cycle arrest and initiating its differentiation program. Interestingly, the $L n c M y o D$ gene is located at about $30 \mathrm{~kb}$ upstream of the mouse $M y o D$ gene and is specifically expressed in myoblasts during early differentiation. LncMyoD deficiency significantly inhibits terminal myoblasts differentiation due to continual cell cycling. $L n c M y o D$ is a $M y o D$ target that can directly bind to the protein IMP2, and compete for IMP binding to inhibit cell cycling and enhance differentiation (Gong et al., 2015). Another lncRNA MUNC is transcribed from about $5 \mathrm{~kb}$ upstream of the MyoD gene. MUNC works as a DRR-enhancer RNA to activate myogenic gene expression (Mueller et al., 2015). Overall, lncRNAs are important regulators for skeletal muscle development.

A set of myogenesis-associated lncRNAs, Yam 1-4, can interact with YY1 and regulate myogenic differentiation ( $\mathrm{Lu} \mathrm{L}$. et al., 2013). The Yam-1 gene is located on chromosome 17 and can bind the $687 \mathrm{bp}$ upstream of the $Y Y 1$ promoter. It is expressed in the nucleus and cytoplasm of myogenic progenitors (Lu L. et al., 2013). Yam-1 can inhibit myogenic differentiation because Yam1 silencing significantly enhances Myogenin, Tnni2, troponin and $\alpha$-actin expression, and it increases myotube formation in mouse myoblasts. Mechanistically, Yam-1 can cis-regulate miR715 expression, which subsequently targets $\mathrm{Wnt7b}$ to impair the Wnt/ $\beta$-catenin signaling. The Yam-2 and Yam-3 are located on mouse chromosome 3 and 15, respectively. Yam-2 and Yam-3 can enhance myogenic differentiation because Yam-2 or Yam-3 deficiency delays the myogenic program by reducing myogenic marker expression. In contrast, Yam-4 silencing enhances myogenic differentiation. Overall, Yam-1 and Yam -4 inhibit myogenesis, while Yam-2 and Yam -3 promote myogenic differentiation. However, the molecular mechanisms by which these lncRNAs regulate myogenic differentiation remain to be further explored.

Metastasis-associated lung adenocarcinoma transcript 1 can regulate skeletal muscle differentiation (Schmidt et al., 2011; Watts et al., 2013) and its expression is highly upregulated in early regeneration stage of satellite cells, but downregulated when the fibers matured and regeneration completed (Chen X. et al., 2017). Malat1 silencing accelerates muscle regeneration and enhances $\mathrm{C} 2 \mathrm{C} 12$ differentiation, while Malat1 overexpression has opposite effects. Further RNA-seq reveals that Malat1 silencing up-regulates the expression of muscle-related genes, but down-regulates the expression of cell cycle-related terms, suggesting that Malat1 inhibits muscle differentiation. Mechanistically, Malat1 suppresses myoblast differentiation as a novel downstream target of myostatin (Watts et al., 2013). Another study indicates that there is a regulatory axis of miR-181a-Malat1-MyoD/Suv39h1 during myogenesis because pro-myogenic miR-181a suppresses Malat1 expression during differentiation (Chen X. et al., 2017). The reduced Malat1 
IncRNA is replaced by activating complex in the $M y o D$ gene locus and subsequently induces MyoD expression and myoblast differentiation (Chen X. et al., 2017). There are other lncRNAs that participate in regulation of myogenesis. Collectively, there are several lncRNAs with diverse functions to regulate skeletal muscle myogenesis. Some of these lncRNAs contribute to the process of skeletal muscle dysfunction and may be potential therapeutic targets (Wang L. et al., 2015).

\section{LncRNA IN NEUROGENESIS}

Neural ESC differentiation is regulated by a complex network and understanding it may help with generating of high-quality neuronal stem cells for disease intervention. Transcriptome profiling analysis has identified many lncRNAs that are differentially expressed during neural differentiation. These indicate that IncRNA can regulate neural differentiation (Mercer et al., 2010).

A genome wide shRNA library targeting 1280 lncRNAs in mouse genome has identified nearly 20 lncRNAs that are essential for neuronal stem cell pluripotency (Lin et al., 2014). TUNA is a pluripotency-associated lncRNA that is exclusively expressed in the CNS. TUNA silencing in ESCs arrests neural differentiation. In mechanism, TUNA can interact and recruit multiprotein complex (PTBP1, hnRNP-K, and nucleolin) to their gene promoters to induce H3K4me, increasing Nanog, Sox2, and Fgf4 activity (Lin et al., 2014). LncRNA-1604 is another lncRNA essential for neural differentiation of mESCs that is predominantly expressed in the brain and striatum. LncRNA-1604 deficiency inhibits ectoderm differentiation and decreases the expression of neural progenitor cell markers (Sox1, Nestin, Zfp521, Pax6, and N-cad). Further luciferase and RIP assays demonstrate that $\operatorname{lncRNA-1604}$ can interact and sponge $m i R-200 c$ to suppress the expression of the core transcription factors ZEB1/2 and the subsequently neural differentiation (Weng et al., 2018).

Maternally expressed gene 3 is derived from the DLK1DIO3 imprinted locus. MEG3 $3^{-/-}$hESCs decreases the neural lineage differentiation rate by downregulating neural lineage marker expression, while induction of MEG3 expression has the opposite effect. Decreases in DLK1-DIO3-imprinted locusinduced lncRNAs can decrease neural lineage differentiation potential (Mo et al., 2015). Evf2 is transcribed from the Dlx5 and Dlx6 intergenic region, and it plays a vital role in early hippocampal development. Evf 2 deficiency reduces the number of GABAergic neurons, impairs synaptic inhibition in the hippocampus and dentate gyrus during early postnatal development (Feng et al., 2006). Mechanistically, the $5^{\prime}$ UCE region of Evf2 can repress the short-range targets Dlx5/6 and the long-range targets Rbm 28 and Akr1b8, while the $3^{\prime}$ UCE of Evf2 can activate the long-range targets Umad1 and Lsm8. Thus, Evf2 regulates neuronal differentiation through ncRNA-dependent topological and transcriptional control (Feng et al., 2006).

Recent microarray analyses reveal that $35 \operatorname{lncRNAs}$ are upregulated during neuronal differentiation of hESCs, including RMST, DalI and PAUPAR (Ng et al., 2012, 2013). RMST is specifically expressed in the brain and crucial for neurogenesis (Ng et al., 2012, 2013). RMST expression is regulated by two neural-specific transcription factors of PAX2 and REST. RMST can bind to SOX2 and is essential for SOX2 binding to the promoter regions of neurogenic transcription factors $(\mathrm{Ng}$ et al., 2012, 2013). While DalI can bind to crucial neurogenic transcription factor, POU3F3, and many of others involved in regulating cell cycling and neuronal development (Chalei et al., 2014). DalI deficiency inhibits neural differentiation in mouse N2A cells. Mechanistically, DalI can target gene expression and DNA methylation via interacting with DNMT1 (DNA methyltransferase) and POU3F3 (Ramos et al., 2016) while PAUPAR can target PAX6 expression (Vance et al., 2014). In contrast, lncRNA Pnky is expressed in human and mouse neural stem cells and its silencing enhances neuronal differentiation, indicating its inhibitory function (Keppetipola et al., 2016). Pnky can interact with PTBP 1 to regulate neuronal differentiationassociated gene transcription (Ramos et al., 2015; Keppetipola et al., 2016). Therefore, there are many lncRNAs that regulate neural differentiation of human and mouse ESCs positively and negatively by targeting various genes. It is important to further investigate how these $\ln c R N A$ s regulate the dynamic neurogenesis process.

\section{LnCRNA IN CARDIOVASCULAR DEVELOPMENT}

Cardiovascular diseases are human health threats due to their high mortality and therapeutic strategy with CPCs has been attractive. However, CPC identification remains challenging. Therefore, it is imperative to understand specific cardiac lineage differentiation (Bruneau, 2013). Recently, emerging evidence uncovers that lncRNAs are crucial for cardiovascular development and maintaining cardiac integrity (Yang et al., 2014; Devaux et al., 2015).

Heart Brake LncRNA 1 is a human-specific lncRNA related to cardiomyocyte differentiation. HBL1 over-expression suppresses cardiomyocyte differentiation by down-regulating GATA4, CTNT, NKX2.5, TBX5, TBX20 and $\alpha$-MHC expression while its silencing has opposite effects (Liu et al., 2017). Mechanistically, $H B L 1$ can interact and sponge $m i R-1$, a key cardiomyocyte differentiation promoter, to impair cardiomyocyte differentiation (Lu T.Y. et al., 2013; Liu et al., 2017). LncRNA Braveheart $(B v h t)$ is exclusively expressed in the heart and can promote cardiovascular lineage commitment of mESCs by activating functional upstream genes of the MesP1, a permissive regulator of multipotent cardiovascular progenitor (Bondue et al., 2008, 2011). Further SHAPE probing and DMS probing assay demonstrate that Bvht secondary structure has a $5^{\prime}$ AGIL and a $11 \mathrm{nt}$ motif in this $5^{\prime}$ AGIL, which is necessary for the cardiac differentiation process and cardiac transcription factor expression. Mechanistically, Bvht can antagonize the cardiac differentiation process negative regulator, cellular $\mathrm{CNBP}$, the zinc finger TF (Xue et al., 2016) and interact with SUZ12 to epigenetically regulate cardiomyocyte differentiation. Hence, lncRNA may regulate gene expression though specific motifs. 
Fendrr is a lateral mesoderm-specific and essential regulator for proper heart development in mice. Fendrr is located upstream of the $5^{\prime}$-end of Foxf1, and it is predominantly localized in the nucleus. Its mutants impair LPM lineages development, induces myocardial dysfunction and may be responsible for embryonic death. Fendrr deficiency down-regulates lateral plate or cardiac mesoderm differentiation-associated transcription factor expression, decreases H3K27 trimethylation, and/or increases in $\mathrm{H} 3 \mathrm{~K} 4$ trimethylation in the PRC2 occupied gene promoters. Mechanistically, Fendrr binds to the Foxf1 and Pitx2 gene promoters and represses the targeted gene expression via increasing PRC2 occupancy and H3K27me3 trimethylation (Grote and Herrmann, 2013; Cekin et al., 2018). In addition, Fendrr may regulate atherosclerosis development (Cekin et al., 2018). CARMEN is a human super enhancer-associated lncRNA with three isoforms that is highly expressed in differentiating CPCs to positively regulate cardiac differentiation. CARMEN deficiency impairs the capacity of human CPCs to differentiate into cardiomyocytes and significantly reduces the expression of cardiac transcription and differentiation makers (GATA4, NKX2.5, TBX5, and others). Therefore, CARMEN may initiate a cardiogenic differentiation program after damage, and it may be a potentially attractive therapeutic target for future regenerative and cell-based therapies (Bruneau, 2013).

A previous RNA-seq study has shown that IncRNAs, TTN-AS1, ALIEN and PUNISHER are differentially expressed in cardiovascular differentiation (Li Y. et al., 2017). TTNAS1 and the TANC1 are highly co-expressed during heart differentiation. TTN-AS1 is regulated by MITF and TBX2, but its function during cardiovascular differentiation has not been explored ( $\mathrm{Li} \mathrm{Y}$. et al., 2017). ALIEN is expressed in the nucleus and perinuclear regions of CPCs, and it can positively regulate cardiovascular development because ALIEN silencing upregulates the expression of genes involved in cell adhesion and extracellular matrix remodeling. However, it downregulates angiogenesis and blood vessel development-associated gene expression. PUNISHER is an antisense transcript of the AGAP2 gene that is expressed in the cytoplasm of differentiated endothelial cells. PUNISHER expression is correlated positively with endothelial cell transcription factor (TAL1 and FOXC1) and vascular development related genes. PUNISHER deficiency downregulates histone $\mathrm{H} 3$ phosphorylation, and impairs human vessel maturation (Kurian et al., 2015).

Recently, Frank et al. (2019) defined a class of divergent lncRNAs in a hESC-based cardiac differentiation model. The so-called yin yang lncRNAs (yylncRNAs) exhibited the same expression pattern as their protein-coding counterparts. Among those lncRNAs, $y y \operatorname{lnc} T$ was identified as a mesodermal commitment specific lncRNA. It was transcribed from the mesoderm specifier BRACHYURY (T) locus and expressed in parallel with T during hESC cardiac commitment. Mechanically, yylncT worked as an essential activator of $\mathrm{T}$ by inhibiting DNMT3B activity to maintain the hypomethylation at the T/yylncT locus (Frank et al., 2019). Collectively, these lncRNAs act as stage-specific regulators of cardiovascular development. Technological development has discovered more and more functional lncRNAs in cardiovascular development. Further exploration will offer us more details about the stage specific regulation of lncRNAs in cardiomyocyte differentiation.

\section{LnCRNA IN EPIDERMOPOIESIS AND HEMATOPOIESIS}

Anti-differentiation ncRNA is preferably expressed in progenitor keratinocytes, but significantly down-regulated in terminally differentiated cells (Kretz et al., 2012). ANCR deficiency reduces keratinocyte differentiation marker expression, including transcription factors that promote differentiation. Mechanistically, ANCR can recruit the PRC2 complex to the epidermal differentiation related $M A F$ and $M A F B$ promoters although ANCR can also promote osteoblast differentiation by up-regulating Runx2 expression (Chen and Zhang, 2016). Transcriptome sequencing reveals that TINCR is one of the most highly induced lncRNAs in the cytoplasm of keratinocyte progenitors during keratinocyte differentiation. TINCR deficiency decreases differentiation-related gene expression and affects the formation of lipid barrier and keratohyalin granules in keratinocytes. PMA and RIA-seq demonstrate that TINCR can bind to mRNA decay protein STAU1, and interact with and stabilize MAF and MAFB mRNAs, as well as CALML5 protein to promote keratinocyte differentiation (Kretz et al., 2013; Lopez-Pajares, 2016). HULC can promote ADSCs differentiation into epithelial and smooth-muscle-like cells. Its overexpression increases the expression of Uroplakin-II, AE1/AE3, $\alpha$-SMA, SM-MHC, Calponin, and SM-22 $\alpha$. Mechanistically, HULC positively regulates BMP9 expression and the $\mathrm{Wnt} / \beta$-catenin activation, but it inactivates the Notch signaling during ADSC differentiation (Li et al., 2018).

Hematopoietic stem cells can differentiate into different types of blood cells. Besides transcription factors and miRNAs, recent studies reveal that lncRNA participates in hematopoiesis, particularly in the myeloid lineage development. The lncRNA $E G O$ is crucial for an eosinophil development (Wagner et al., 2007). EGO silencing impairs the expression of basic protein and neurotoxin that are essential for eosinophil development, suggesting that EGO can positively regulate eosinophil lineage differentiation (Wagner et al., 2007). LncRNA also regulates the granulocyte and monocyte formation process. HOTAIRM1 is a human HoxA cluster transcribed antisense lncRNA that is highly expressed in myeloid progenitor cells upon granulocytic differentiation. HOTAIRM1 silencing inhibits HoxA1, HoxA4, CD11b and CD18 expression, impairing myeloid cell differentiation (Zhang et al., 2009). LncRNA HoxBlinc is a specific marker for Flk1 + mesoderm that can promote hematopoietic differentiation. Mesoderm-derived Flk1 + cells can be induced to be cardiogenic and hemangiogenic progenitors. HoxBlinc knockout downregulates the expression of $H o x B$ gene and other cardiac/hematopoietic differentiation related genes and the components of the Notch signaling. In mechanism, HoxBlinc can bind to the HoxB genes and activated HoxB gene expression through recruiting the Setdla/MLL1 complex and mediating long-range chromatin interactions. 


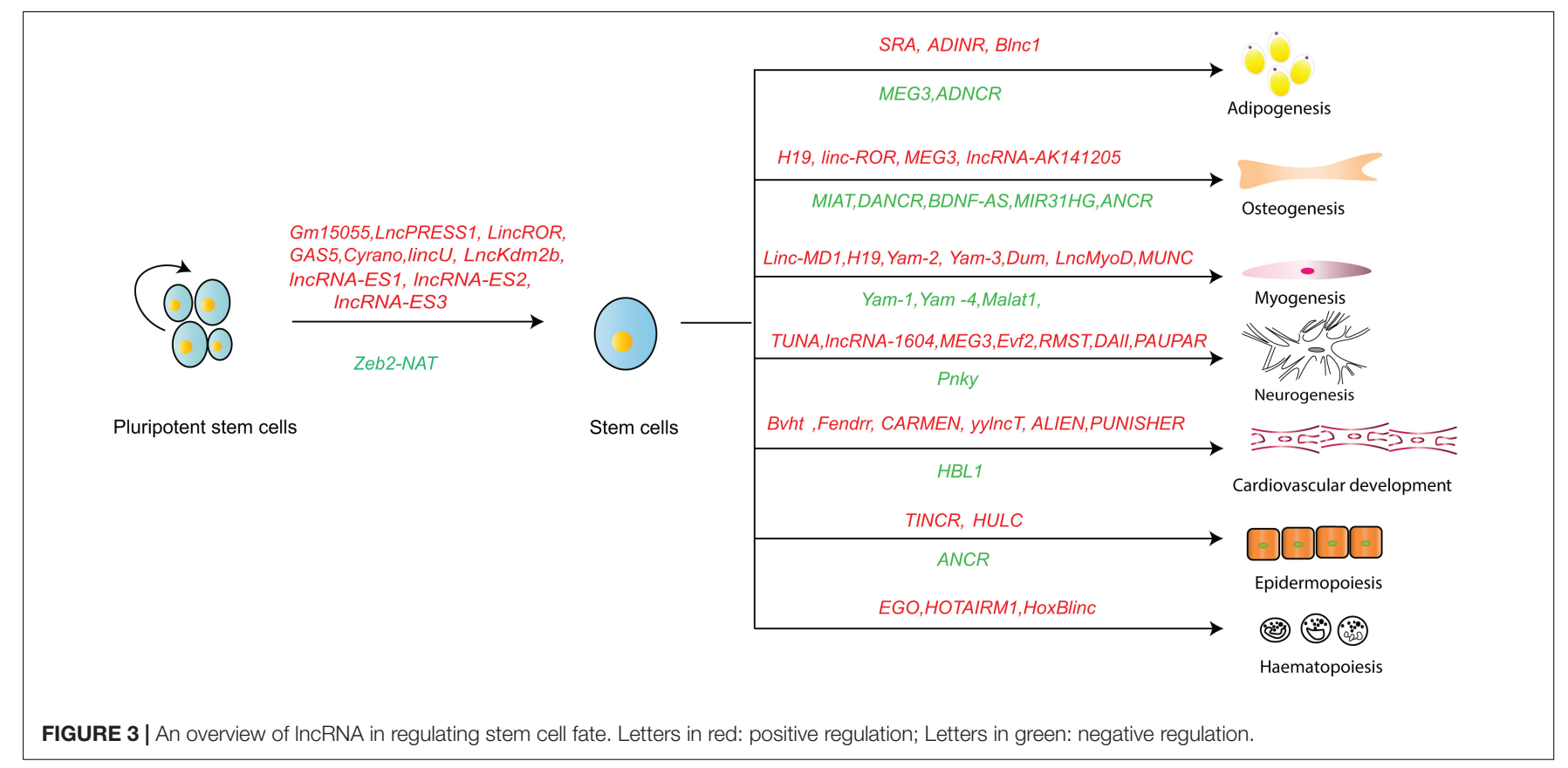

HoxBlinc can also regulate the Wnt/Notch signaling and Hox pathways (Deng et al., 2016).

\section{CONCLUSION AND FURTHER PERSPECTIVE}

As a major part of mammal genome, lncRNAs play essential roles in determining stem cell fates via different mechanisms. Some lncRNAs may be involved in different biological processes. For example, lncROR may maintain stem cell pluripotency (Wang et al., 2013) and promote the osteogenic differentiation process (Feng L. et al., 2018). MEG3 is a positive regulator of both osteogenesis (Zhuang et al., 2015) and neurogenesis (Mo et al., 2015), while it acts as an adipogenesis inhibitor (Li Z. et al., 2017). LncRNA regulation presents a complex molecular interaction network. Some pluripotent reprograming regulatory lncRNAs have been recently identified using CRIST-seq (Zhang et al., 2019). They form a regulatory network around the Oct4 and Sox2 promoters and affect reprograming through epigenetic pathways, including coordination of intrachromosomal loops, alteration of the methylation levels, and activation of the eRNA pathway for stemness genes (Jia et al., 2020; Wang et al., 2020). It is interesting to explore whether these lncRNAs work in coordination, and what their spatial and temporal specialties are in regulating the fate of stem cells.

The current understanding of lncRNA is more comprehensive due to improved technologies for lncRNA functionality, structure and interacting partners. LncRNA is important player in stem cell pluripotency maintenance, differentiation and their dysfunction-related human diseases through different mechanisms. The recently discovered lncRNAs that maintain stem cell pluripotency and determine their lineage differentiation are summarized in Figure 3. Interestingly, besides sequence conservation, the RNA structure and the location of IncRNAs seem to be good indicators for their function. Since lncRNA regulate somatic cell reprograming and stem cell differentiation, the manipulation of lncRNA may develop better protocols for efficient somatic cell reprograming and stem cell differentiation. There are new approaches to understand lncRNA regulation. For example, RAT-seq enables identification of the genome-wide chromatin binding sites for a specific lncRNA (Chen et al., 2018; Du et al., 2018). CRIST-seq could identify lncRNAs within the regulatory elements of stemness genes (Zhang et al., 2019). RIP, crosslinking and immunoprecipitation (CLIP), and RNA pull-down techniques are used for identifying the interaction proteomes of lncRNAs (Cao et al., 2019). These approaches will uncover more important roles and new mechanistic insights through which lncRNAs regulate biological process and relevant diseases. Conceivably, these new insights may also reveal new therapeutic targets and aid in design of new therapies for human diseases (Dey et al., 2014a).

\section{AUTHOR CONTRIBUTIONS}

JC, YW, and CW collected the data. JC wrote the manuscript. J$\mathrm{FH}$ and WL provided guidelines and edited the manuscript. All authors read and approved the final manuscript.

\section{FUNDING}

This work was supported by the National Key R\&D Program of China (2018YFA0106902) and National Key Research and Development Program of China grant (2016YFC13038000). 


\section{REFERENCES}

Batista, P. J., and Chang, H. Y. (2013). Long noncoding RNAs: cellular address codes in development and disease. Cell 152, 1298-1307. doi: 10.1016/j.cell.2013. 02.012

Bernardes de Jesus, B., Marinho, S. P., Barros, S., Sousa-Franco, A., Alves-Vale, C., Carvalho, T., et al. (2018). Silencing of the lncRNA Zeb2-NAT facilitates reprogramming of aged fibroblasts and safeguards stem cell pluripotency. Nat. Commun. 9:94. doi: 10.1038/s41467-017-01921-6

Bondue, A., Lapouge, G., Paulissen, C., Semeraro, C., Iacovino, M., Kyba, M., et al. (2008). Mesp1 acts as a master regulator of multipotent cardiovascular progenitor specification. Cell Stem Cell 3, 69-84. doi: 10.1016/j.stem.2008. 06.009

Bondue, A., Tannler, S., Chiapparo, G., Chabab, S., Ramialison, M., Paulissen, C., et al. (2011). Defining the earliest step of cardiovascular progenitor specification during embryonic stem cell differentiation. J. Cell Biol. 192, 751-765. doi: 10 1083/jcb.201007063

Borsos, M., and Torres-Padilla, M. E. (2016). Building up the nucleus: nuclear organization in the establishment of totipotency and pluripotency during mammalian development. Genes Dev. 30, 611-621. doi: 10.1101/gad.273 805.115

Bruneau, B. G. (2013). Signaling and transcriptional networks in heart development and regeneration. Cold Spring Harb. Perspect. Biol. 5:a008292. doi: 10.1101/cshperspect.a008292

Cao, J. (2014). The functional role of long non-coding RNAs and epigenetics. Biol. Proced. Online 16:11. doi: 10.1186/1480-9222-16-11

Cao, M., Zhao, J., and Hu, G. (2019). Genome-wide methods for investigating long noncoding RNAs. Biomed. Pharmacother. 111, 395-401. doi: 10.1016/j.biopha. 2018.12.078

Cekin, N., Ozcan, A., Goksel, S., Arslan, S., Pinarbasi, E., and Berkan, O. (2018). Decreased FENDRR and LincRNA-p21 expression in atherosclerotic plaque. Anatol. J. Cardiol. 19, 131-136. doi: 10.14744/AnatolJCardiol.2017.8081

Cesana, M., Cacchiarelli, D., Legnini, I., Santini, T., Sthandier, O., Chinappi, M., et al. (2011). A long noncoding RNA controls muscle differentiation by functioning as a competing endogenous RNA. Cell 147, 358-369. doi: 10.1016/ j.cell.2011.09.028

Chalei, V., Sansom, S. N., Kong, L., Lee, S., Montiel, J. F., Vance, K. W., et al. (2014). The long non-coding RNA Dali is an epigenetic regulator of neural differentiation. eLife 3:e04530. doi: 10.7554/eLife.04530

Chen, C. Y., Cheng, Y. Y., Yen, C. Y., and Hsieh, P. C. (2017). Mechanisms of pluripotency maintenance in mouse embryonic stem cells. Cell. Mol. Life Sci. 74, 1805-1817. doi: 10.1007/s00018-016-2438-0

Chen, L., and Zhang, S. (2016). Long noncoding RNAs in cell differentiation and pluripotency. Cell Tissue Res. 366, 509-521. doi: 10.1007/s00441-016-2451-5

Chen, N., Zhao, G., Yan, X., Lv, Z., Yin, H., Zhang, S., et al. (2018). A novel FLI1 exonic circular RNA promotes metastasis in breast cancer by coordinately regulating TET1 and DNMT1. Genome Biol. 19:218. doi: 10.1186/s13059-0181594-y

Chen, X., He, L., Zhao, Y., Li, Y., Zhang, S., Sun, K., et al. (2017). Malat1 regulates myogenic differentiation and muscle regeneration through modulating MyoD transcriptional activity. Cell Discov. 3:17002. doi: 10.1038/celldisc.2017.2

Deng, C., Li, Y., Zhou, L., Cho, J., Patel, B., Terada, N., et al. (2016). HoxBlinc RNA recruits Set1/MLL complexes to activate Hox gene expression patterns and mesoderm lineage development. Cell Rep. 14, 103-114. doi: 10.1016/j.celrep. 2015.12.007

Devaux, Y., Zangrando, J., Schroen, B., Creemers, E. E., Pedrazzini, T., Chang, C. P., et al. (2015). Long noncoding RNAs in cardiac development and ageing. Nat. Rev. Cardiol. 12, 415-425. doi: 10.1038/nrcardio.2015.55

Dey, B. K., Mueller, A. C., and Dutta, A. (2014a). Long non-coding RNAs as emerging regulators of differentiation, development, and disease. Transcription 5:e944014. doi: 10.4161/21541272.2014.944014

Dey, B. K., Pfeifer, K., and Dutta, A. (2014b). The H19 long noncoding RNA gives rise to microRNAs miR-675-3p and miR-675-5p to promote skeletal muscle differentiation and regeneration. Genes Dev. 28, 491-501. doi: 10.1101/gad. 234419.113

Du, Z., Jia, L., Wang, Y., Wang, C., Wen, X., Chen, J., et al. (2018). Combined RNA-seq and RAT-seq mapping of long noncoding RNAs in pluripotent reprogramming. Sci. Data 5:180255. doi: 10.1038/sdata.2018.255
Feng, J., Bi, C., Clark, B. S., Mady, R., Shah, P., and Kohtz, J. D. (2006). The Evf-2 noncoding RNA is transcribed from the Dlx-5/6 ultraconserved region and functions as a Dlx-2 transcriptional coactivator. Genes Dev. 20, 1470-1484. doi: 10.1101/gad.1416106

Feng, L., Shi, L., Lu, Y. F., Wang, B., Tang, T., Fu, W. M., et al. (2018). Linc-ROR promotes osteogenic differentiation of mesenchymal stem cells by functioning as a competing endogenous RNA for miR-138 and miR-145. Mol. Ther. Nucleic Acids 11, 345-353. doi: 10.1016/j.omtn.2018.03.004

Feng, X., Lin, T., Liu, X., Yang, C., Yang, S., and Fu, D. (2018). Long non-coding RNA BDNF-AS modulates osteogenic differentiation of bone marrow-derived mesenchymal stem cells. Mol. Cell. Biochem. 445, 59-65. doi: 10.1007/s11010017-3251-2

Fico, A., Fiorenzano, A., Pascale, E., Patriarca, E. J., and Minchiotti, G. (2019). Long non-coding RNA in stem cell pluripotency and lineage commitment: functions and evolutionary conservation. Cell. Mol. Life Sci. 76, 1459-1471. doi: 10.1007/s00018-018-3000-z

Frank, S., Ahuja, G., Bartsch, D., Russ, N., Yao, W., Kuo, J. C., et al. (2019). yylncT defines a class of divergently transcribed lncRNAs and safeguards the t-mediated mesodermal commitment of human PSCs. Cell Stem Cell 24, 318-327.e8. doi: 10.1016/j.stem.2018.11.005

Frankish, A., Diekhans, M., Ferreira, A. M., Johnson, R., Jungreis, I., Loveland, J., et al. (2019). GENCODE reference annotation for the human and mouse genomes. Nucleic Acids Res. 47, D766-D773. doi: 10.1093/nar/gky955

Gong, C., Li, Z., Ramanujan, K., Clay, I., Zhang, Y., Lemire-Brachat, S., et al. (2015). A long non-coding RNA, LncMyoD, regulates skeletal muscle differentiation by blocking IMP2-mediated mRNA translation. Dev. Cell 34, 181-191. doi: 10.1016/j.devcel.2015.05.009

Grote, P., and Herrmann, B. G. (2013). The long non-coding RNA Fendrr links epigenetic control mechanisms to gene regulatory networks in mammalian embryogenesis. RNA Biol. 10, 1579-1585. doi: 10.4161/rna.26165

Guttman, M., Donaghey, J., Carey, B. W., Garber, M., Grenier, J. K., Munson, G., et al. (2011). lincRNAs act in the circuitry controlling pluripotency and differentiation. Nature 477, 295-300. doi: 10.1038/nature10398

Hassani, S. N., Moradi, S., Taleahmad, S., Braun, T., and Baharvand, H. (2019). Transition of inner cell mass to embryonic stem cells: mechanisms, facts, and hypotheses. Cell. Mol. Life Sci. 76, 873-892. doi: 10.1007/s00018-018-2965-y

$\mathrm{Hu}$, J. F., and Hoffman, A. R. (2014). Chromatin looping is needed for iPSC induction. Cell Cycle 13, 1-2. doi: 10.4161/cc.27017

Jain, A. K., Xi, Y., McCarthy, R., Allton, K., Akdemir, K. C., Patel, L. R., et al. (2016). LncPRESS1 is a p53-regulated LncRNA that safeguards pluripotency by disrupting SIRT6-mediated de-acetylation of histone H3K56. Mol. Cell 64, 967-981. doi: 10.1016/j.molcel.2016.10.039

Jia, L., Wang, Y., Wang, C., Du, Z., Zhang, S., Wen, X., et al. (2020). Oplr16 serves as a novel chromatin factor to control stem cell fate by modulating pluripotencyspecific chromosomal looping and TET2-mediated DNA demethylation. Nucleic Acids Res. 48:gkaa097. doi: 10.1093/nar/gkaa097

Jiapaer, Z., Li, G., Ye, D., Bai, M., Li, J., Guo, X., et al. (2018). LincU preserves naive pluripotency by restricting ERK activity in embryonic stem cells. Stem Cell Rep. 11, 395-409. doi: 10.1016/j.stemcr.2018.06.010

Jin, C., Jia, L., Huang, Y., Zheng, Y., Du, N., Liu, Y., et al. (2016). Inhibition of lncRNA MIR31HG promotes osteogenic differentiation of human adiposederived stem cells. Stem Cells 34, 2707-2720. doi: 10.1002/stem.2439

Jin, C., Zheng, Y., Huang, Y., Liu, Y., Jia, L., and Zhou, Y. (2017). Long noncoding RNA MIAT knockdown promotes osteogenic differentiation of human adipose-derived stem cells. Cell Biol. Int. 41, 33-41. doi: 10.1002/cbin.10697

Kallen, A. N., Zhou, X. B., Xu, J., Qiao, C., Ma, J., Yan, L., et al. (2013). The imprinted H19 lncRNA antagonizes let-7 microRNAs. Mol. Cell 52, 101-112. doi: 10.1016/j.molcel.2013.08.027

Keppetipola, N. M., Yeom, K. H., Hernandez, A. L., Bui, T., Sharma, S., and Black, D. L. (2016). Multiple determinants of splicing repression activity in the polypyrimidine tract binding proteins, PTBP1 and PTBP2. RNA 22, 1172-1180. doi: 10.1261/rna.057505.116

Kretz, M., Siprashvili, Z., Chu, C., Webster, D. E., Zehnder, A., Qu, K., et al. (2013). Control of somatic tissue differentiation by the long non-coding RNA TINCR. Nature 493, 231-235. doi: 10.1038/nature11661

Kretz, M., Webster, D. E., Flockhart, R. J., Lee, C. S., Zehnder, A., Lopez-Pajares, V., et al. (2012). Suppression of progenitor differentiation requires the long noncoding RNA ANCR. Genes Dev. 26, 338-343. doi: 10.1101/gad.182121.111 
Kung, J. T., Colognori, D., and Lee, J. T. (2013). Long noncoding RNAs: past, present, and future. Genetics 193, 651-669. doi: 10.1534/genetics.112.146704

Kurian, L., Aguirre, A., Sancho-Martinez, I., Benner, C., Hishida, T., Nguyen, T. B., et al. (2015). Identification of novel long noncoding RNAs underlying vertebrate cardiovascular development. Circulation 131, 1278-1290. doi: 10. 1161/circulationaha.114.013303

Lanz, R. B., Chua, S. S., Barron, N., Soder, B. M., DeMayo, F., and O'Malley, B. W. (2003). Steroid receptor RNA activator stimulates proliferation as well as apoptosis in vivo. Mol. Cell Biol. 23, 7163-7176. doi: 10.1128/mcb.23.20.71637176.2003

Li, M., Sun, X., Cai, H., Sun, Y., Plath, M., Li, C., et al. (2016). Long non-coding RNA ADNCR suppresses adipogenic differentiation by targeting miR-204. Biochim. Biophys. Acta 1859, 871-882. doi: 10.1016/j.bbagrm.2016.05.003

Li, Y., Shan, Z., Yang, B., Yang, D., Men, C., Cui, Y., et al. (2018). LncRNA HULC promotes epithelial and smooth-muscle-like differentiation of adipose-derived stem cells by upregulation of BMP9. Pharmazie 73, 49-55. doi: 10.1691/ph.2018. 7634

Li, Y., Zhang, J., Huo, C., Ding, N., Li, J., Xiao, J., et al. (2017). Dynamic organization of lncRNA and circular RNA regulators collectively controlled cardiac differentiation in humans. EBiomedicine 24, 137-146. doi: 10.1016/j. ebiom.2017.09.015

Li, Z., Jin, C., Chen, S., Zheng, Y., Huang, Y., Jia, L., et al. (2017). Long noncoding RNA MEG3 inhibits adipogenesis and promotes osteogenesis of human adipose-derived mesenchymal stem cells via miR-140-5p. Mol. Cell. Biochem. 433, 51-60. doi: 10.1007/s11010-017-3015-z

Liang, W. C., Fu, W. M., Wang, Y. B., Sun, Y. X., Xu, L. L., Wong, C. W., et al. (2016). H19 activates Wnt signaling and promotes osteoblast differentiation by functioning as a competing endogenous RNA. Sci. Rep. 6:20121. doi: 10.1038/ srep20121

Lin, N., Chang, K. Y., Li, Z., Gates, K., Rana, Z. A., Dang, J., et al. (2014). An evolutionarily conserved long noncoding RNA TUNA controls pluripotency and neural lineage commitment. Mol. Cell 53, 1005-1019. doi: 10.1016/j.molcel. 2014.01.021

Liu, G. Y., Zhao, G. N., Chen, X. F., Hao, D. L., Zhao, X., Lv, X., et al. (2016). The long noncoding RNA Gm15055 represses Hoxa gene expression by recruiting PRC2 to the gene cluster. Nucleic Acids Res. 44, 2613-2627. doi: 10.1093/nar/ gkv1315

Liu, J., Li, Y., Lin, B., Sheng, Y., and Yang, L. (2017). HBL1 is a human long noncoding RNA that modulates cardiomyocyte development from pluripotent stem cells by counteracting MIR1. Dev. Cell 42, 333-348.e5. doi: 10.1016/j. devcel.2017.07.023

Loewer, S., Cabili, M. N., Guttman, M., Loh, Y. H., Thomas, K., Park, I. H., et al. (2010). Large intergenic non-coding RNA-RoR modulates reprogramming of human induced pluripotent stem cells. Nat. Genet. 42, 1113-1117. doi: 10.1038/ ng.710

Long, F. (2011). Building strong bones: molecular regulation of the osteoblast lineage. Nat. Rev. Mol. Cell Biol. 13, 27-38. doi: 10.1038/nrm3254

Lopez-Pajares, V. (2016). Long non-coding RNA regulation of gene expression during differentiation. Pflugers Arch. 468, 971-981. doi: 10.1007/s00424-0161809-6

Lu, L., Sun, K., Chen, X., Zhao, Y., Wang, L., Zhou, L., et al. (2013). Genome-wide survey by ChIP-seq reveals YY1 regulation of lincRNAs in skeletal myogenesis. EMBO J. 32, 2575-2588. doi: 10.1038/emboj.2013.182

Lu, T. Y., Lin, B., Li, Y., Arora, A., Han, L., Cui, C., et al. (2013). Overexpression of microRNA-1 promotes cardiomyocyte commitment from human cardiovascular progenitors via suppressing WNT and FGF signaling pathways. J. Mol. Cell. Cardiol. 63, 146-154. doi: 10.1016/j.yjmcc.2013.07.019

Mercer, T. R., Dinger, M. E., and Mattick, J. S. (2009). Long non-coding RNAs: insights into functions. Nat. Rev. Genet. 10, 155-159. doi: 10.1038/nrg2521

Mercer, T. R., Qureshi, I. A., Gokhan, S., Dinger, M. E., Li, G., Mattick, J. S., et al. (2010). Long noncoding RNAs in neuronal-glial fate specification and oligodendrocyte lineage maturation. BMC Neurosci. 11:14. doi: 10.1186/14712202-11-14

Mo, C. F., Wu, F. C., Tai, K. Y., Chang, W. C., Chang, K. W., Kuo, H. C., et al. (2015). Loss of non-coding RNA expression from the DLK1-DIO3 imprinted locus correlates with reduced neural differentiation potential in human embryonic stem cell lines. Stem Cell Res. Ther. 6:1. doi: 10.1186/scrt535
Mueller, A. C., Cichewicz, M. A., Dey, B. K., Layer, R., Reon, B. J., Gagan, J. R., et al. (2015). MUNC, a long noncoding RNA that facilitates the function of MyoD in skeletal myogenesis. Mol. Cell Biol. 35, 498-513. doi: 10.1128/mcb.01079-14

Ng, S. Y., Bogu, G. K., Soh, B. S., and Stanton, L. W. (2013). The long noncoding RNA RMST interacts with SOX2 to regulate neurogenesis. Mol. Cell 51, 349359. doi: 10.1016/j.molcel.2013.07.017

Ng, S. Y., Johnson, R., and Stanton, L. W. (2012). Human long non-coding RNAs promote pluripotency and neuronal differentiation by association with chromatin modifiers and transcription factors. $E M B O J .31,522-533$. doi: 10. 1038/emboj.2011.459

Pisignano, G., Pavlaki, I., and Murrell, A. (2019). Being in a loop: how long noncoding RNAs organise genome architecture. Essays Biochem. 63, 177-186. doi: $10.1042 /$ ebc20180057

Ponting, C. P., Oliver, P. L., and Reik, W. (2009). Evolution and functions of long noncoding RNAs. Cell 136, 629-641. doi: 10.1016/j.cell.2009.02.006

Quinn, J. J., and Chang, H. Y. (2016). Unique features of long non-coding RNA biogenesis and function. Nat. Rev. Genet. 17, 47-62. doi: 10.1038/nrg.2015.10

Ramos, A. D., Andersen, R. E., Liu, S. J., Nowakowski, T. J., Hong, S. J., Gertz, C., et al. (2015). The long noncoding RNA Pnky regulates neuronal differentiation of embryonic and postnatal neural stem cells. Cell Stem Cell 16, 439-447. doi: 10.1016/j.stem.2015.02.007

Ramos, A. D., Attenello, F. J., and Lim, D. A. (2016). Uncovering the roles of long noncoding RNAs in neural development and glioma progression. Neurosci. Lett. 625, 70-79. doi: 10.1016/j.neulet.2015.12.025

Rosa, A., and Ballarino, M. (2016). Long noncoding RNA regulation of pluripotency. Stem Cells Int. 2016:1797692. doi: 10.1155/2016/1797692

Salmena, L., Poliseno, L., Tay, Y., Kats, L., and Pandolfi, P. P. (2011). A ceRNA hypothesis: the Rosetta Stone of a hidden RNA language? Cell 146, 353-358. doi: 10.1016/j.cell.2011.07.014

Schmidt, L. H., Spieker, T., Koschmieder, S., Schaffers, S., Humberg, J., Jungen, D., et al. (2011). The long noncoding MALAT-1 RNA indicates a poor prognosis in non-small cell lung cancer and induces migration and tumor growth. J. Thorac. Oncol. 6, 1984-1992. doi: 10.1097/JTO.0b013e3182307eac

Shen, Y., Dong, L. F., Zhou, R. M., Yao, J., Song, Y. C., Yang, H., et al. (2016). Role of long non-coding RNA MIAT in proliferation, apoptosis and migration of lens epithelial cells: a clinical and in vitro study. J. Cell. Mol. Med. 20, 537-548. doi: $10.1111 /$ jcmm. 12755

Sheng, L., Ye, L., Zhang, D., Cawthorn, W. P., and Xu, B. (2018). New insights into the long non-coding RNA SRA: physiological functions and mechanisms of action. Front. Med. 5:244. doi: 10.3389/fmed.2018.00244

Smith, K. N., Starmer, J., Miller, S. C., Sethupathy, P., and Magnuson, T. (2017). Long noncoding RNA moderates MicroRNA activity to maintain self-renewal in embryonic stem cells. Stem Cell Rep. 9, 108-121. doi: 10.1016/j.stemcr.2017. 05.005

Takahashi, K., and Yamanaka, S. (2006). Induction of pluripotent stem cells from mouse embryonic and adult fibroblast cultures by defined factors. Cell 126, 663-676. doi: 10.1016/j.cell.2006.07.024

Thomson, J. A., Itskovitz-Eldor, J., Shapiro, S. S., Waknitz, M. A., Swiergiel, J. J., Marshall, V. S., et al. (1998). Embryonic stem cell lines derived from human blastocysts. Science 282, 1145-1147. doi: 10.1126/science.282.5391.1145

Tye, C. E., Gordon, J. A., Martin-Buley, L. A., Stein, J. L., Lian, J. B., and Stein, G. S. (2015). Could lncRNAs be the missing links in control of mesenchymal stem cell differentiation? J. Cell. Physiol. 230, 526-534. doi: 10.1002/jcp.24834

Vance, K. W., Sansom, S. N., Lee, S., Chalei, V., Kong, L., Cooper, S. E., et al. (2014). The long non-coding RNA Paupar regulates the expression of both local and distal genes. EMBO J. 33, 296-311. doi: 10.1002/embj.201386225

Wagner, L. A., Christensen, C. J., Dunn, D. M., Spangrude, G. J., Georgelas, A., Kelley, L., et al. (2007). EGO, a novel, noncoding RNA gene, regulates eosinophil granule protein transcript expression. Blood 109, 5191-5198. doi: 10.1182/blood-2006-06-027987

Wang, C., Jia, L., Wang, Y., Du, Z., Zhou, L., Wen, X., et al. (2020). Genome-wide interaction target profiling reveals a novel Peblr20-eRNA activation pathway to control stem cell pluripotency. Theranostics 10, 353-370. doi: 10.7150/thno. 39093

Wang, H., Ge, S., Qian, G., Li, W., Cui, J., Wang, G., et al. (2015). Restoration of IGF2 imprinting by polycomb repressive complex 2 docking factor SUZ12 in colon cancer cells. Exp. Cell Res. 338, 214-221. doi: 10.1016/j.yexcr.2015.09.016 
Wang, L., Zhao, Y., Bao, X., Zhu, X., Kwok, Y. K., Sun, K., et al. (2015). LncRNA Dum interacts with Dnmts to regulate Dppa2 expression during myogenic differentiation and muscle regeneration. Cell Res. 25, 335-350. doi: 10.1038/cr. 2015.21

Wang, K. C., and Chang, H. Y. (2011). Molecular mechanisms of long noncoding RNAs. Mol. Cell 43, 904-914. doi: 10.1016/j.molcel.2011.08.018

Wang, Y., Xu, Z., Jiang, J., Xu, C., Kang, J., Xiao, L., et al. (2013). Endogenous miRNA sponge lincRNA-RoR regulates Oct4, Nanog, and Sox2 in human embryonic stem cell self-renewal. Dev. Cell 25, 69-80. doi: 10.1016/j.devcel. 2013.03.002

Wapinski, O., and Chang, H. Y. (2011). Long noncoding RNAs and human disease. Trends Cell Biol. 21, 354-361. doi: 10.1016/j.tcb.2011.04.001

Watts, R., Johnsen, V. L., Shearer, J., and Hittel, D. S. (2013). Myostatin-induced inhibition of the long noncoding RNA Malat1 is associated with decreased myogenesis. Am. J. Physiol. Cell. Physiol. 304, C995-C1001. doi: 10.1152/ajpcell. 00392.2012

Weng, R., Lu, C., Liu, X., Li, G., Lan, Y., Qiao, J., et al. (2018). Long noncoding RNA-1604 orchestrates neural differentiation through the miR-200c/ZEB axis. Stem Cells 36, 325-336. doi: 10.1002/stem.2749

Xiao, T., Liu, L., Li, H., Sun, Y., Luo, H., Li, T., et al. (2015). Long noncoding RNA ADINR regulates adipogenesis by transcriptionally activating C/EBPalpha. Stem Cell Rep. 5, 856-865. doi: 10.1016/j.stemcr.2015.09.007

Xu, C., Zhang, Y., Wang, Q., Xu, Z., Jiang, J., Gao, Y., et al. (2016). Long non-coding RNA GAS5 controls human embryonic stem cell self-renewal by maintaining NODAL signalling. Nat. Commun. 7:13287. doi: 10.1038/ncomms13287

Xu, X., Ji, S., Li, W., Yi, B., Li, H., Zhang, H., et al. (2017). LncRNA H19 promotes the differentiation of bovine skeletal muscle satellite cells by suppressing Sirt1/FoxO1. Cell. Mol. Biol. Lett. 22:10. doi: 10.1186/s11658-017-0040-6

Xue, Z., Hennelly, S., Doyle, B., Gulati, A. A., Novikova, I. V., Sanbonmatsu, K. Y., et al. (2016). A G-Rich motif in the lncRNA braveheart interacts with a zincfinger transcription factor to specify the cardiovascular lineage. Mol. Cell 64, 37-50. doi: 10.1016/j.molcel.2016.08.010

Yang, J. H., Li, J. H., Jiang, S., Zhou, H., and Qu, L. H. (2013). ChIPBase: a database for decoding the transcriptional regulation of long non-coding RNA and microRNA genes from ChIP-Seq data. Nucleic Acids Res. 41, D177-D187. doi: 10.1093/nar/gks1060

Yang, K. C., Yamada, K. A., Patel, A. Y., Topkara, V. K., George, I., Cheema, F. H., et al. (2014). Deep RNA sequencing reveals dynamic regulation of myocardial noncoding RNAs in failing human heart and remodeling with mechanical circulatory support. Circulation 29, 1009-1021. doi: 10.1161/circulationaha.113. 003863

Ye, B., Liu, B., Yang, L., Zhu, X., Zhang, D., Wu, W., et al. (2018). LncKdm2b controls self-renewal of embryonic stem cells via activating expression of transcription factor Zbtb3. EMBO J. 37:e97174. doi: 10.15252/embj.201797174
Yu, J., Vodyanik, M. A., Smuga-Otto, K., Antosiewicz-Bourget, J., Frane, J. L., Tian, S., et al. (2007). Induced pluripotent stem cell lines derived from human somatic cells. Science 318, 1917-1920. doi: 10.1126/science.1151526

Zhang, H., Jiao, W., Sun, L., Fan, J., Chen, M., Wang, H., et al. (2013). Intrachromosomal looping is required for activation of endogenous pluripotency genes during reprogramming. Cell Stem Cell 13, 30-35. doi: 10.1016/j.stem.2013.05.012

Zhang, H., Zeitz, M. J., Wang, H., Niu, B., Ge, S., Li, W., et al. (2014). Long noncoding RNA-mediated intrachromosomal interactions promote imprinting at the Kcnq1 locus. J. Cell Biol. 204, 61-75. doi: 10.1083/jcb.201304152

Zhang, J., Tao, Z., and Wang, Y. (2018). Long noncoding RNA DANCR regulates the proliferation and osteogenic differentiation of human bone-derived marrow mesenchymal stem cells via the p38 MAPK pathway. Int. J. Mol. Med. 41, 213-219. doi: 10.3892/ijmm.2017.3215

Zhang, S., Wang, Y., Jia, L., Wen, X., Du, Z., Wang, C., et al. (2019). Profiling the long noncoding RNA interaction network in the regulatory elements of target genes by chromatin in situ reverse transcription sequencing. Genome Res. 29, 1521-1532. doi: 10.1101/gr.244996.118

Zhang, X., Lian, Z., Padden, C., Gerstein, M. B., Rozowsky, J., Snyder, M., et al. (2009). A myelopoiesis-associated regulatory intergenic noncoding RNA transcript within the human HOXA cluster. Blood 113, 2526-2534. doi: 10. 1182/blood-2008-06-162164

Zhao, X. Y., Li, S., Wang, G. X., Yu, Q., and Lin, J. D. (2014). A long noncoding RNA transcriptional regulatory circuit drives thermogenic adipocyte differentiation. Mol. Cell 55, 372-382. doi: 10.1016/j.molcel.2014. 06.004

Zhu, L., and Xu, P. C. (2013). Downregulated LncRNA-ANCR promotes osteoblast differentiation by targeting EZH2 and regulating Runx2 expression. Biochem. Biophys. Res. Commun. 432, 612-617. doi: 10.1016/j.bbrc.2013.02.036

Zhuang, W., Ge, X., Yang, S., Huang, M., Zhuang, W., Chen, P., et al. (2015). Upregulation of lncRNA MEG3 promotes osteogenic differentiation of mesenchymal stem cells from multiple myeloma patients by targeting BMP4 transcription. Stem Cells 33, 1985-1997. doi: 10.1002/stem.1989

Conflict of Interest: The authors declare that the research was conducted in the absence of any commercial or financial relationships that could be construed as a potential conflict of interest.

Copyright (c) 2020 Chen, Wang, Wang, Hu and Li. This is an open-access article distributed under the terms of the Creative Commons Attribution License (CC BY). The use, distribution or reproduction in other forums is permitted, provided the original author(s) and the copyright owner(s) are credited and that the original publication in this journal is cited, in accordance with accepted academic practice. No use, distribution or reproduction is permitted which does not comply with these terms. 